\title{
Study of Prevalence of Metformin Associated Lactic Acidosis (MALA) In Patients with Creatinine Clearance More Than 20ml/Min/Per $1.73 \mathrm{~m}^{2}$
}

\author{
Dr Gomathy $S^{1}$,Dr Usha Samuel ${ }^{2}$, Dr Balaprabhan $\mathrm{G}^{3}$,Dr Legha $\mathrm{R}^{4}$ \\ ${ }^{I}$ (Additional Professor, Dept Of Nephrology, Govt.T.D. Medical College, Alappuzha, India ) \\ ${ }^{2}$ (Professor Dept Of Nephrology, Govt.T .D. Medical College, Alappuzha, India) \\ 3 (Junior Resident, Department of Internal Medicine, Govt.T.D. Medical College, Alappuzha, India) \\ ${ }^{4}$ (Professor, Department of Internal Medicine, Govt.T.D. Medical College, Alappuzha, India)
}

\begin{abstract}
A common clinical challenge facing all practitioners treating patients with type 2 diabetes mellitus today are the guidelines for metformin contraindicating its use in men and women with serum creatnine concentrations $\geq 1.5$ and $\geq 1.4 \mathrm{mg} / \mathrm{dl}(\geq 132$ and $\geq 123 \mathrm{mmol} / \mathrm{L})$,respectively. In a patient tolerating and controlled with this medication should it automatically be discontinued as the creatnine rises beyond these cut points over time? Stopping metformin often results in poorly controlled glycemia. Despite the proven benefits ,metformin remains contraindicated in a large segment of the type 2 diabetic population,largely because of concerns over the rare adverse effect of lactic acidosis. For these reasons, the drug has been restricted to individuals with normal creatnine levels as a surrogate for renal competence. How many patients are unable to receive this medication on the basis of guidelines which, although well intentioned, are somewhat arbitrary and outdated based on modern assessments of renal status? Given the current contraindications, we might consider it a challenge to conduct a new clinical trial to evaluate the use of metformin in individuals with various degrees of impaired renal function,taking into account new criteria for assessing glomerular filtration,attempting to translate creatnine into corresponding eGFR cut points in the context of metformin therapy. The present study was conducted in patients with type 2 diabetes mellitus who attended medical OP or admitted in medical wards in Govt.T.D.Medical college, Alappuzha in age group 13 years and more old during the period March 2014 to Feb 2015. The ultimate aim was to find the prevalence of lactic acidosis in patients taking metformin with creatnine clearance more than $20 \mathrm{ml} / \mathrm{min}$.

Objective: To study the prevalence of lactic acidosis in patient taking metformin with creatnine clearance more than $20 \mathrm{ml} /$ minute/per $1.73 \mathrm{~m}^{2}$.

Methodology: The study was conducted under the Department of nephrology,Government T D Medical college,Alappuzha which is a teritiary care hospital in central Kerala,South India.Patients attending the department of General Medicine and Nephrology who satisfied the inclusion criteria were enrolled in the study .All Type 2 DM patients with creatinine clearance above $20 \mathrm{ml} /$ minute and on metformin monotherapy and age group of 13 years and above were included.

Observations and conclusions: Out of the 102 patients studied, 65 were males and 37 were females. 8 of them were $<40$ years, 62 were between 40-60 years age group and 32 were more than 60 years age group. The data analysed showed four cases of metabolic acidosis.The bicarbonate levels were low 21.8,21.0,21.4,21.5 mmol/L and the eGFR values were 22.50,28.56,23.33 and $31.27 \mathrm{ml} / \mathrm{min}$ per $\mathrm{m}^{2}$ respectively.Based on our study findings, Metformin use is cautioned and very close monitoring is required when the eGFR falls to $<30$ and $\geq 20 \mathrm{ml} / \mathrm{min}$ per $1.73 \mathrm{~m}^{2}$ and to avoid Metformin use if eGFR $<20 \mathrm{ml} / \mathrm{min}$ per $1.73 \mathrm{~m}^{2}$.

Keywords: : Diabetes, Metformin, Metabolic Acidosis.
\end{abstract}

\section{Introduction}

Metformin is the only biguanide available in U.S .Another biguanide ,phenformin has been used since the 1950's,but was declared an " imminent hazard " in 1976 because of lactic acidosis. At the time of its removal from the market there had been 306 documented cases of phenformin-associated lactic acidosis,including 1 fatal and 2 nonfatal cases in the randomized control trial of the university group diabetes program. Metformin was marketed as Glucophage by Bristol-Myers Squibb in early 1995, with a boxed warning concerning the risk of lactic acidosis. Metformin had been used widely in Europe for several years, where it had been recognized that the riskof lactic acidosis from metformin was no greater than the risk of hypoglycemia from sulfonylureas. Although the clinical utility of metformin had been recognized in the U.S ,its approval was undoubtedlydelayed by the specter of phenformin and the lingering concern that metformin might also cause lactic acidosis. The eventual approval of metformin was unusuallycontroversial. Crofford predicted that metformin "will be widely used and will improve the outlook for many patients" with diabetes. But fear of lactic acidosis led the consumer advocacy group public citizen to issue the warning " Do not use Glucophage". ${ }^{1}$

DOI: 10.9790/0853-1606035159 $\quad$ www.iosrjournals.org $51 \mid$ Page


Based on the data from Sweden, it was estimated that the risk of lactic acidosis in patients taking phenformin was 10fold higher than the risk in patients taking metformin. A later report from Sweden concluded that discontinuing metformin in aging patients when they developed renal or cardiovascular disease could further reduce the risk of lactic acidosis. The labeling of Glucophage reflected a belief that metformin had the potential to cause lactic acidosis, but that the risk could be mitigated by careful selection of patients.

Metformin rarely, if ever,causes lactic acidosis when it is used as labeled. Metformin is associated with lactic acidosis in patients with conditions that can themselves cause lactic acidosis (heart failure,hypoxia,sepsis,etc). But it is impossible to determine to what extent,if any, metformin may contribute to development of lactic acidosis in any individual case. When metformin is used as labeled, the increased risk of lactic acidosis is either zero or so close to zero that it cannot be factored into ordinary clinical decision making. That metformin can itself cause lactic acidosis is supported by the finding of lactic acidosis in people who took overdoses. Thus, the accumulation of metformin in the setting of renal insufficiency might be expected to precipitate lactic acidosis in some patients who are at risk. If one excludes overdoses, most cases of metformin - associated lactic acidosis,particularly the fatal ones, were probably not caused by metformin.

Estimated 2-10 patients per 1,00,000 patients receiving metformin develops lactic acidosis per year. Metformin associated lactic acidosis accounts for approximately0.1-1\% of total patients admitted to ICU. Life threatening condition with mortality rate of 30-50\%. Nearly all of the reported cases of metformin associated lactic acidosis have occurred in individuals with both hemodynamic(e.g. hypotension) and metabolic (e.g.liver or renal disease) causes of impaired lactic metabolism. Controversy remains whether the use of metformin is a cause or a coincidence in lactic acidosis of diabetes mellitus patients. ${ }^{2}$

\section{Metformin}

Metformin is an oral antidiabetic drug widely prescribed as the first line drug of choice for the treatment of type 2 diabetes mellitus,in particular in overweightand obese people and those with normal kidney function. Metformin belongs to the biguanide class of antidiabetic drugs. The term "biguanide" often refers specifically to a class of drugs that function as oral anti hyperglycemic drugs used for diabetes mellitus or prediabetes treatment.

Metformin is a type 2 diabetic drug and contains the active ingredient metformin hydrochloride. Metformin helps to lower blood glucose levels by reducing the amount of glucose produced and released by the liver and by increasing insulin sensitivity. Metformin is usually taken at meal times however your doctor will advise you when and how often to take metformin .Metformin may be available in tablet form or in sachets of powder. Metformin can be used in conjunction with other diabetic drugs or as a single treatment (a monotherapy) and diabetics should also use diet and exercise to help control their condition.

The drug helps in type 2 diabetes mellitus respond better to their own insulin,decreases hyperglycemia primarily by suppressing glucose production by the liver(hepatic gluconeogenesis). Metformin treatment reduces this by over one third. In addition to suppressing hepatic glucose production,metformin increases insulin sensitivity,enhances peripheral glucose uptake(by inducing the phosphorylation of GLUT4 enhancer factor), decreases insulin induced suppression of fatty acid oxidation and decreases absorption of glucose from the gastrointestinal tract.

The molecular mechanism of metfromin is incompletely understood. Inhibition of the mitochondrial respiratory chain complex 1 , activation of AMP-activated protein kinase(AMPK), inhibition of glucagon induced elevation of cyclic adenosine monophosphate(cAMP) and consequent activation of protein kinase A(PKA) , inhibition of mitochondrial glycerophosphate dehydrogenase and an effect on gut microbiota have been proposed as potential mechanisms.

Metformin is primarily used for type 2 diabetes, but is increasingly being used in polycystic ovary syndrome. Certain evidences suggests metformin may prevent the cardiovascular and possibly the cancer complications of diabetes. It helps reduce LDL cholesterol and triglyceride levels and is not associated with weight gain; in some people,it promotes weight loss. Metformin is one of only two oral antidiabetics in world health organization model list of essential medicines(the other being Glibenclamide).

\section{Lactic Acidosis}

Lactic acidosis is a form of metabolic acidosis due to the inadequate clearance of lactic acid from theblood. Lactate is a byproduct of anaerobic respiration and is normally cleared from the blood by the liver,kidney and skeletal muscle. Lactic acidosis occurs when the body's buffering systems are overloaded and tends to cause a $\mathrm{pH}$ of $\leq 7.25$ with plasma lactate $\geq 5 \mathrm{mmol} / \mathrm{L}$.It is usually caused by a state of tissue hypoperfusion and/or hypoxia.This causes pyruvic acid to be preferentially converted to lactate during anaerobic respiration. Hyperlactatemia is defined as plasma lactate $>2 \mathrm{mmol} / \mathrm{L}$ 
Causes

- Enhance metabolic rate (eg:seizures and exercise)

- Reduced oxygen delivery (eg:cardiac or pulmonary failure,shock,tissue infarction)

- Diminished lactate catabolism due to hepatic failure or hypoperfusion

- Decreased oxygen utilization (eg;cyanide poisoning)

- Enzymatic defects in glycenolysis or gluconeogenesis.

\section{Classification of Lactic Acidosis}

TYPE A: Lactic acidosis with clinical evidence of tissue hypoperfusion or hypoxia.

TYPE B: Lactic acidosis occurs without clinical evidence of tissue hypoperfusion or hypoxia. It is further subdivided into :

TYPE B1: due to underlying disease (eg; sepsis,chronic kidney disease, heart failure, diabetes, acute severe viral illness,malaria,cholera)

TYPE B2: due to effects of drugs or toxins (eg; biguanides, paracetamol, antoconvulsants, alcohol, aspirin, antituberculous drugs, ethylene glycolpoisoning, sorbitol, sodiumitroprusside, lactulose, theophylline, cocaine, amphetamines, papaverine, paraldehyde)

TYPE B3: due to inborn or acquired errors of metabolism(eg;organic acidemias,primary lactic acidosis,thiamine deficiency,glycogen storage disorders,mitochondrial disease i.e;MELAS-mitochondrial encephalopathy with lactic acidosis and stroke,D-lactic acidosis in short gut syndrome,overexertion,prolonged generalized seizures.[cohen and woods devised the above mentioned classification in 1976 and it is still widely used]

\section{Metformin Associated Lactic Acidosis(Mala)}

Metformin is one of the most commonly prescribed medications for the treatment oftype 2 diabetes mellitus. The relationship between metforminand lactic acidosis is complex,since use of the drug maybe causal,co responsible or coincidental. ${ }^{12}$. Main objective of this article is to counter the conventional view of the link between metformin and lactic acidosis, according to the metformin associated lactic acidosis is rare but is still associated with a high rate of mortality. In fact the direct metformin related mortality is close to zero and metformin may even be protective in cases of very severe lactic acidosis unrelated to the drug. Metformin has also inherited a negative class effect since the early biguanide phenformin was associated with more frequent and sometimes fatal lactic acidosis.

Secondly ,the objective is to identify the most efficient patient management methods based on our knowledge of how metformin acts on glucose/lactate metabolism and how lactic acidosis may occur (at the organ and cellular levels) ${ }^{3}$ during metformin treatment. The liver appears to be a key organ for both the antidiabetic effect of metformin and the development of lactic acidosis. Metformin exerts its activity by increasing peripheral glucose uptake and utilization and decreasing hepatic gluconeogenesis. Lactate uptake by the liver is diminished with metformin administration because lactate is a substrate for hepatic gluconeogenesis.a process which metformin inhibits. By decreasing pyruvate dehydrogenase activity and mitochondrial reducing agent transport, metformin enhances anaerobic metabolism and increased production of trocarboxylic acid cycle precursors. Inhibition of puruvate dehydrogenase subsequently decreases the channeling of these precursors into aerobic metabolism and causes increased metabolism of pyruvate to lactate which effluxes into the circulation rather than being oxidized further and ultimately lactic acid production. Hemodialysis should systematically be performed in severe forms of lactic acidosis,since it provides both symptomatic and etiological treatment (by eliminating lactate and metformin) ${ }^{3}$.

In a patient with normal renalfunction, the excess lactic acid is simply cleared through kidneys and so significant elevation in blood levels of lactate occurs. However, in a patient with renal impairment ,both metformin and lactic acid are cleared less effectively and may result in further accumulation of both and possibly causing a buildup of lactic acid. The complication of lactic acidosis is serious and potentially fatal. Because metformin decreases liver uptake of lactate,any condition that may precipitate lactic acidosis is condraindicated to its use.

Risk factors for lactic acidosis include any disease state that has the potential to either increase the production or decrease the removal of lactic acid. Conditions that may increase the risk include congestive heart failure,liver disease, shock, alcohol use(due to depletion of NAD+ stores).hypoxic states, respiratory disease(due to inadequate oxygenation of tissues),renal failure,sepsis and advanced age. The use of metformin in patients with renal impairment is associated with an increased risk for lactic acidosis.

Increased risk for lactic acidosis associated with metformin is controversial.The complication of lactic acidosis purely by the usage of metformin is very rare and the vast majority of these cases seem to be related to comorbid condition as mentioned above rather than to the metformin itself. 
It appears that metformin itself maynot increase the risk for lactic acidosis. Although metformin use has increased over the years the incidenceof lactic acidosis has not increased. Additionally there is no evidence to suggest that sulfonylureas alone increase the risk for lactic acidosis. Rather the presence of underlying conditions and concomitant use of other medications may elevate an individual's risk level.

A Cochrane systematic review of over 200 trials evaluated the incidence of lactic acidosis among patients prescribed metformin vs non metformin antidiabetic medications. Of 100,000 people ,the incidence of lactic acidosis was 5.1 cases in the metformin group and 5.8 cases in the no-metformin group. The authors concluded that metformin is not associated with an increased risk for lactic acidosis.

To compare the occurrence of lactic acidosis between metformin and sulfonylureas,Bodmer and colleagues conducted a nested case controlled analysis of 50,048 patients with type 2 diabetics receiving an oral antidiabetic agent.They found that 100,000 patients the crude incidence of lactic acidosis in patients treated with metformin was 3.3, while the incidence of lactic acidosis in patients treated with sulfonylureas was 4.8. All patients in whom lactic acidosis developed had underlying conditions that are known risk factors for lactic acidosis.A few of some reviews concluded no data exists todefinitely link metformin to lactic acidosis.

Metformin has also been suggested to increase production of lactate in the small intestine; this could potentially contribute to lactic acidosis in those with risk factors.But the clinical significance of this is unknown and moreover the risk of metformin associated lactic acidosis is most commonly attributed to decreased hepatic uptake rather than intestinal production.

However in practice it may be important to consider diabetes mellitus as a risk factor for developing lactic acidosis and examine each patients's medication profile as well as other underlying conditions to determine a patients's overall risk.

\section{Optimal Treatment Of Metformin Associated Lactic Acidosis(Mala)}

- Is controversial and relies on nonspecific supportive measures

- Gastrointestinal decontamination for acute ingestions; activated charcoal

- Securing airway,circulation and breathing

- For patients with profound acidosis $(\mathrm{pH}<7.10)$ consider sodium bicarbonate infusion eg.1-2 meq/kg intravenous preparation, then 3 ampoule bicarbonate preparation in 1L D5W at $250 \mathrm{ml} / \mathrm{hr}$

- For patients with profound acidosis,renal disease or critical illness consider intermittent hemodialysis/CRRT

- Hemodialysis will correct metformin associated acid base disturbance and increase metformin clearance.

- Sequential measurements of plasma metformin levels during hemodialysis showed that in $85 \%$ of patients a cumulative hemodialysis durationof 15 hours was associated with the return of metformin level to therapeutic values.

\section{Contraindications for Use of Metformin}

- Renal impairment : plasma creatnine values $1.5 \mathrm{mg} / \mathrm{dl}(132 \mathrm{mmol} / \mathrm{l})$ for men and $1.4 \mathrm{mg} / \mathrm{dl}(124 \mathrm{mmol} / \mathrm{l})$ for women.

- Cardiac or respiratory insufficiency that is likely to cause central hypoxia or reduced peripheral perfusion.

- History of lactic acidosis

- Severe infection that could lead to decreased tissue perfusion

- Liver disease including alcoholic liver disease as demonstrated byabnormalliverfunction test.

- Alcohol abuse with binge drinking sufficient to cause acute hepatic toxicity.

- Use of intravenous radiographic contrast agents.

\section{Aims of the study}

1.To study the prevalence of lactic acidosis in patient taking metformin with creatnine clearance more than $20 \mathrm{ml} / \mathrm{min}$ per $1.73 \mathrm{~m}^{2}$

\section{Methodology}

The study was conducted under the Department of nephrology,Government $T$ D Medical college,Alappuzha which is a teritiary care hospital in central Kerala,South India.Patients attending the department of General Medicine and Nephrology who satisfied the inclusion criteria were enrolled in the study during the study period of one year(March 2014 to February 2015). Study was conducted in the cross sectional design.All Type 2 DM patients with creatnine clearance above $20 \mathrm{ml} /$ minute in the age group of 13 years and above were included after taking informed written consent. 


\section{III.1 Inclusion criteria}

Type 2 diabetes mellitus patients proven by FBS,PPBS,HbA1c levels in patients with creatnine clearance above $20 \mathrm{ml} / \mathrm{min}$ are included in our study

\section{III.2 Exclusion criteria}

1.Chronic infection like tuberculosis

2.Chronic kidney disease patients with creatnine clearance below $20 \mathrm{ml} / \mathrm{min}$

3.Chronic liver disease

4.Drug intake which alters renal function test

5.Patients who are not giving consent to participate in the study

Basic clinical details were collected from all the patients and relevant blood investigations like renal function test ,liver function tests, ABG were done.

All the investigations were done in the central laboratory attached to Government T D Medical college Alappuzha.

Blood urea was estimated using GLDH Urease method.Serum creatnine wasestimated using Jaffe's method using alkaline picrate reaction.Urine albumin done by sulphosalicylic acid test.Urine sugar done by Benedict test and deposits are examined by direct microscopy. Bicarbonate levels by using ABG was used as surrogate marker for lactic acidosis.

Statistical analysis was done with SPSS version 16.

\section{Observations and Analysis.}

102 patients were included in the study of which 65 were male patients and 37 were female patients. $60.8 \%$ of them were in the age group of 41 to 60 years.

Age distribution of these patients is given in table 1 .

Table 1. AGE Distribution of patients on Metformin

\begin{tabular}{|l|l|l|l|l|}
\hline Age & Frequency & Percent & Valid percent & Cumulative percent \\
\hline Age $<40$ years & 8 & 7.8 & 7.8 & 7.8 \\
\hline Age between 41-60 years & 62 & 60.8 & 60.8 & 68.6 \\
\hline Age $>60$ years & 32 & 31.4 & 31.4 & 100 \\
\hline Total & 102 & 100 & 100 & \\
\hline
\end{tabular}

(7.8\% was under 40 years; $60.8 \%$ was more than 40 but less than 60 years;31.4\% was more than 60 years). Gender distribution is given in table 2 .

Table 2: Gender distribution of patients on Metformin

\begin{tabular}{|l|l|l|l|l|}
\hline Gender & Frequency & Percent & Valid percent & Cumulative percent \\
\hline Male & 65 & 63.7 & 63.7 & 63.7 \\
\hline Female & 37 & 36.3 & 36.3 & 100 \\
\hline Total & 102 & 100 & 100 & \\
\hline
\end{tabular}

Fig .1 Bicarbonate level distribution of patients on metformin

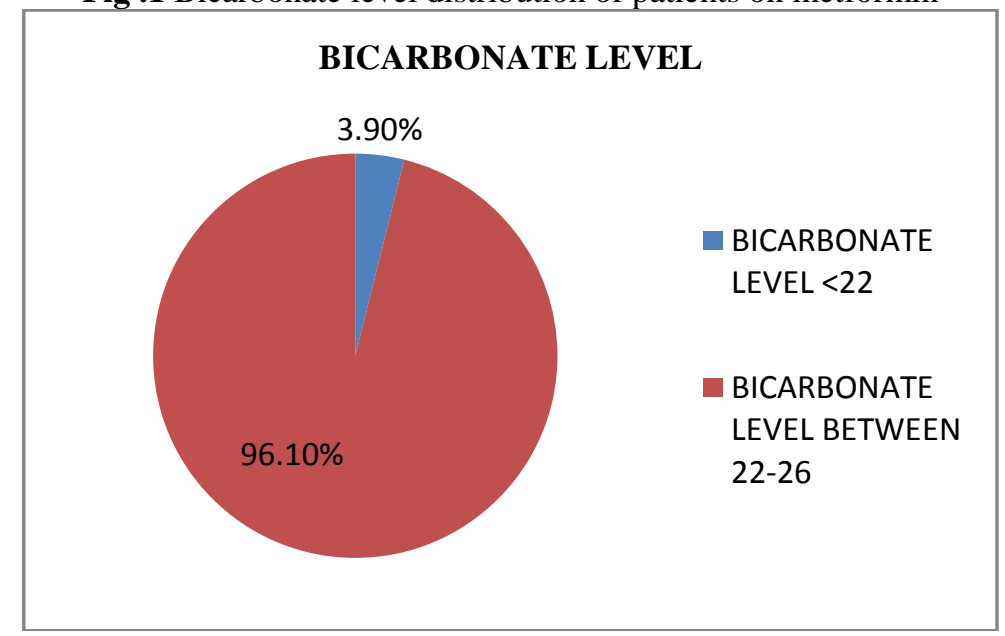

(3.92\% of the patients are with bicarbonate level 22 and $96.8 \%$ between $22-26$ ) 
Fig .2 pH level distribution of patients on metformin

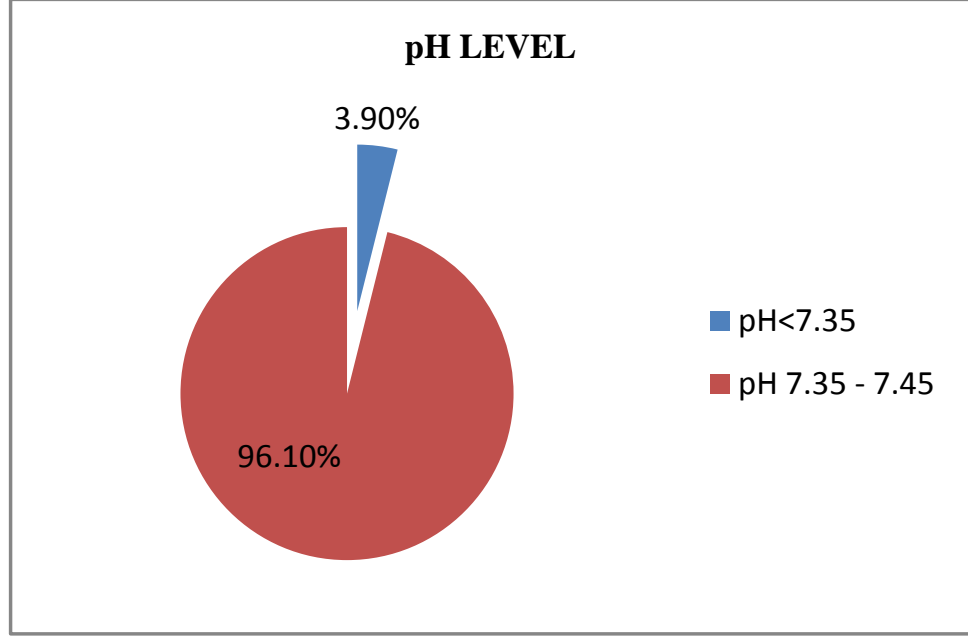

(3.92\% of the patients are $\mathrm{pH}$ below 7.35 and $96.08 \%$ are $\mathrm{pH}$ between $7.35-7.45$ )

Fig .3 Distribution of GFR of patients on Metformin

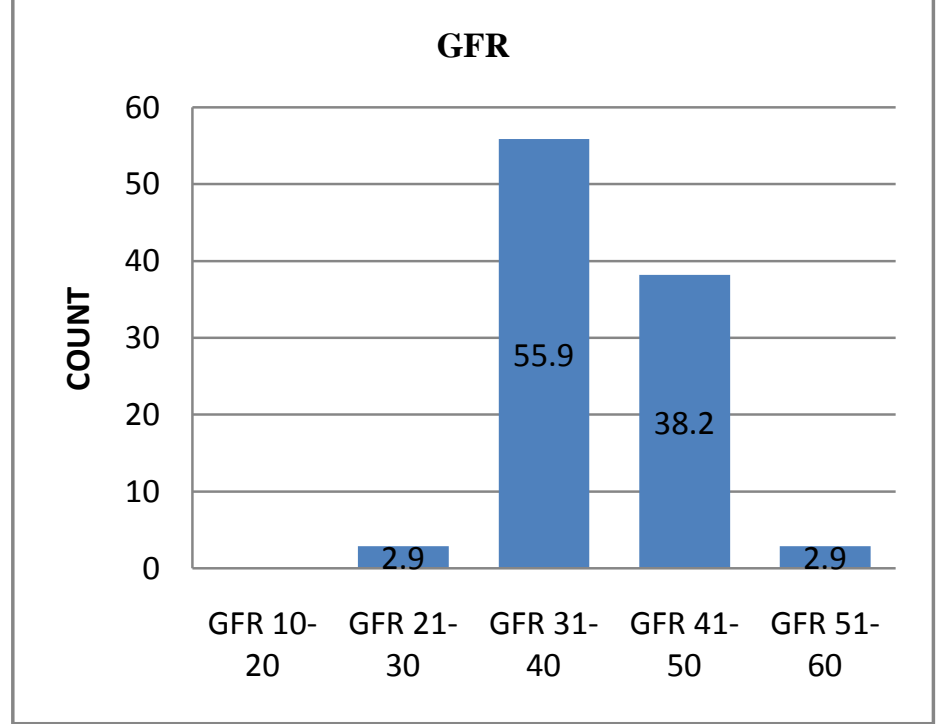

Fig . 4 Bicarbonate /pH distribution in a given GFR Bicarbonate level $<22$ meq/L

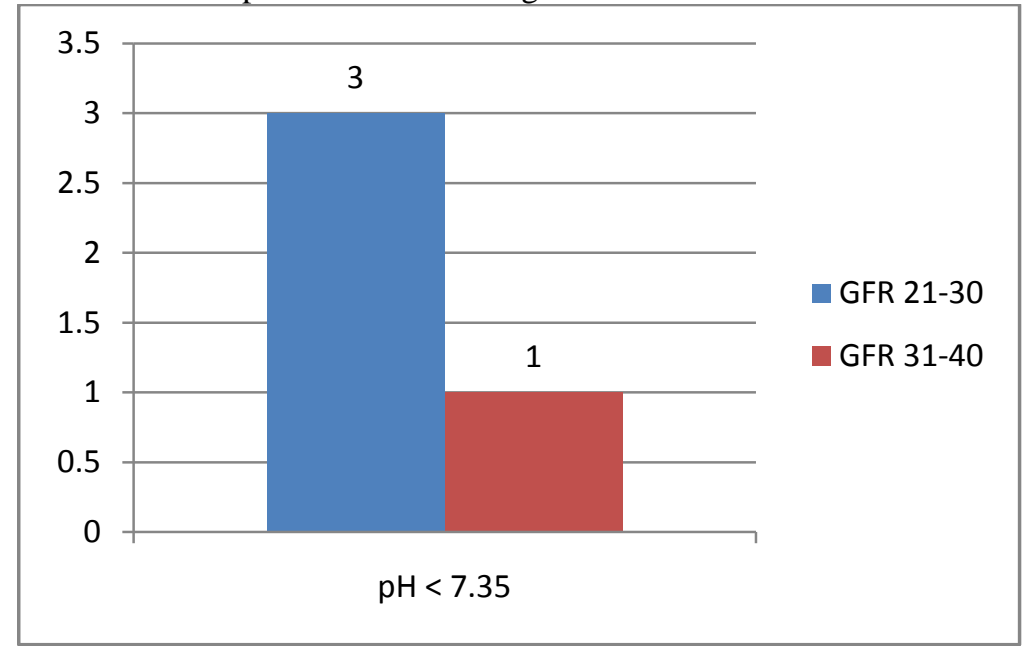


Study of prevalence of metformin associated lactic acidosis(MALA) in patients with creatinine clearance more ..

Fig .5 Bicarbonate /pH distribution in a given GFR Bicarbonate level between 22-26 meq/L

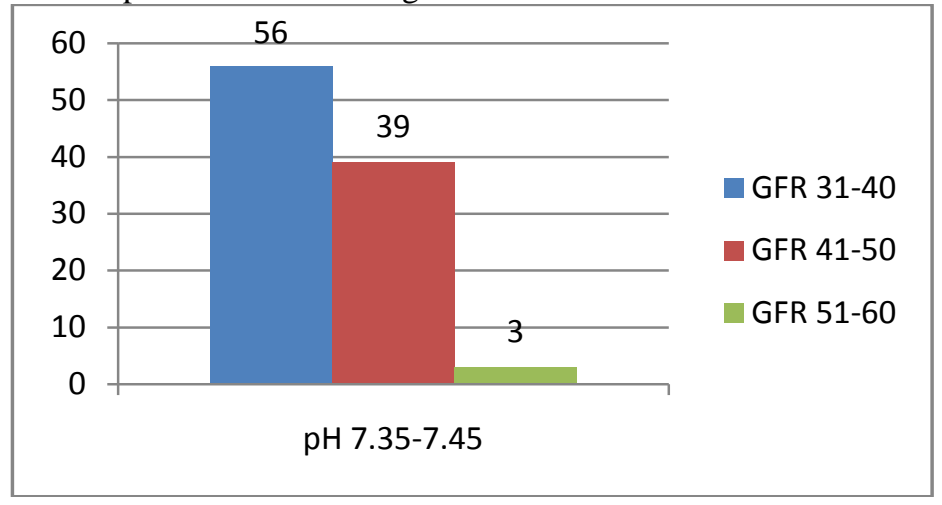

Fig. $6 \mathrm{pH} /$ Bicarbonate distribution in a given GFR range $(\mathrm{pH}<7.35)$

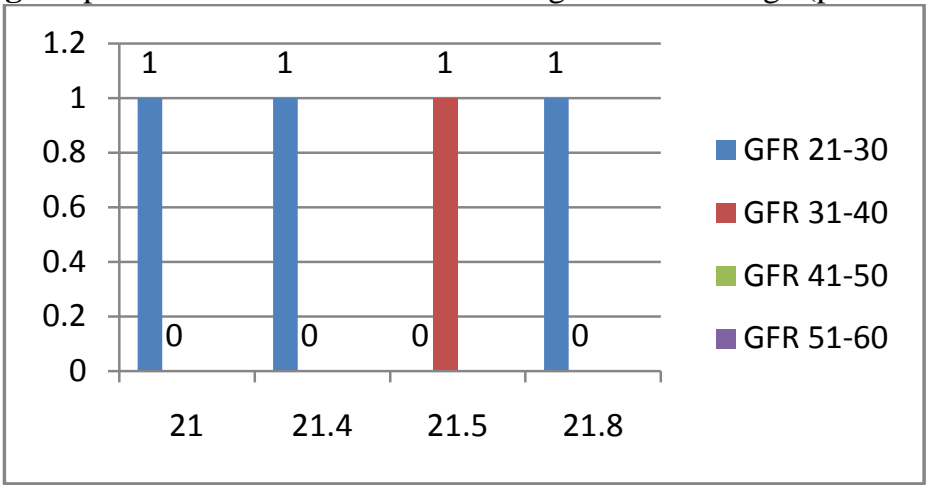

(Chi-square is 4.000; df-3;p-value $=0.261$ )

Fig.7 pH/Bicarbonate distribution ina given GFR range ( $\mathrm{pH}$ between 7.35 -7.45)

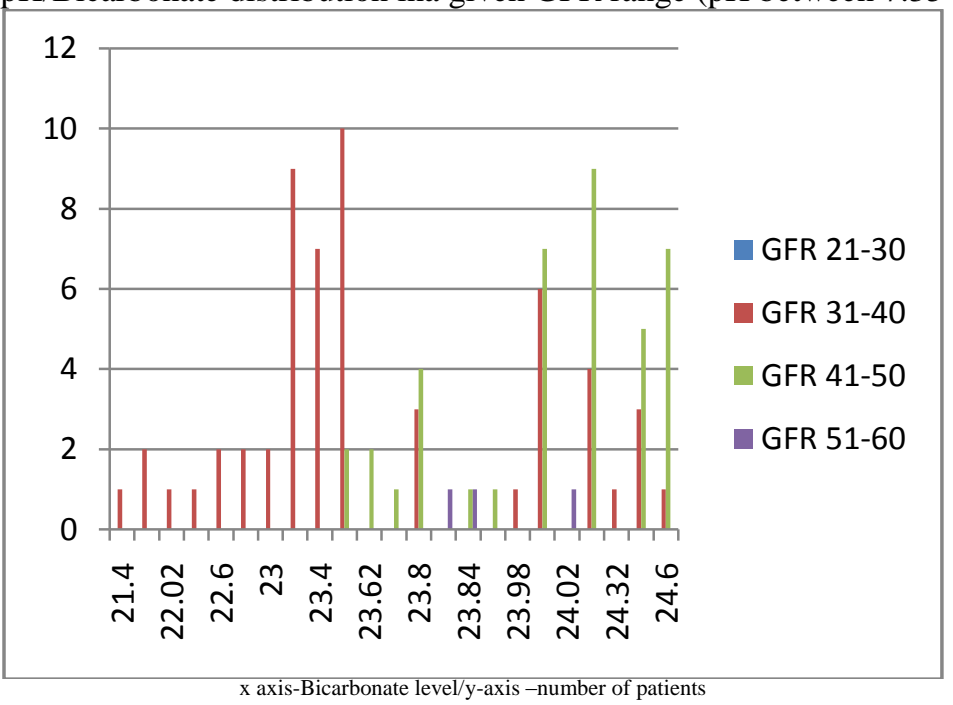

Table.3 Prevalence of acidosis in patients on metformin in various GFR

\begin{tabular}{|l|l|l|l|}
\hline GFR(ml/min) & ACIDOSIS PRESENT & ACIDOSIS ABSENT & TOTAL \\
\hline GFR 21-30 & 3 & 0 & 3 \\
\hline GFR 31-40 & 1 & 56 & 57 \\
\hline GFR 41-50 & 0 & 39 & 39 \\
\hline GFR 51-60 & 0 & 3 & 3 \\
\hline TOTAL & 4 & 98 & 102 \\
\hline
\end{tabular}




\section{Discussions}

Unlike sulfonyl ureas ,thiazolidinediones and insulin ,metformin is weight neutral which makes it an attractive choice for obese patients. Furthermore the management of type 2 diabetes mellitus can be complicated by hypoglycemia which can seriously limit the pursuit of glycemic control ${ }^{4}$.Here too metformin has advantages over insulin and some types of insulin secretagogues;by decreasing excess hepatic gluconeogenesis without raising insulin levels, it rarely leads to significant hypoglycemia when used as a monotherapy.As a result metfromin is widely considered an ideal first lineagent for the treatment of type 2 diabetes as recommended by several guidelines ${ }^{[18,19]}$

In addition to such benefits , metformin reduces the risk of developing diabetes in individuals at high risk for the disease and has been considered as a reasonable "off- label"approach in selected individuals for diabetes prevention ${ }^{[20]}$ Despite these proven benefits , metformin remains contraindicated in a large segment of the type 2 diabetic population,largelybecause of concerns over the rare adverse effect of lactic acidosis. For these reasons, the drug has been restricted to individuals with normal creatnine levels as a surrogate for renal competence. $^{[4,5]}$

Indeed in the modern era of eGFR this measure of glomerular filtration appears to give a more reliable estimate of renal dysfunction. An evidence based approach to prescribing metformin in this group appears warranted taking into account the current pervasive use of eGFR in clinical care. ${ }^{[6]}$ At present the exact glomerular filtrationrate cutoff threshold for the safe use of metformin is controversial.

Clinical guidelines outside of the U.S incorporate the eGFR for determination of metformin safety. In U.K for example prescribing guidelines consider both creatnine and eGFR for assessing eligibility.The National Institute for Health and Clinical Excellence recommends reviewing the clinical circumstances when serum creatnine exceeds $130 \mu \mathrm{mol} / \mathrm{L}(1.5 \mathrm{mg} / \mathrm{dl})$ or eGFR falls below $45 \mathrm{ml} / \mathrm{min}$ per $1.73 \mathrm{~m}^{2}$. The National Institute for Health and Clinical Excellence further specifies that metformin be stopped if serum creatnine exceeds $150 \mu \mathrm{mol} / \mathrm{L}(1.7 \mathrm{mg} / \mathrm{dl})$.a higher threshold than in U.S or eGFR is below $30 \mathrm{ml} / \mathrm{min}$ per $1.73 \mathrm{~m}^{2}{ }^{[6]}$.In contrast, the Canadian diabetes association practice guidelines are now based solely on eGFR,recommending caution with eGFR $<60 \mathrm{ml} / \mathrm{min}$ per $1.73 \mathrm{~m}^{2}$ and contraindicating its use with eGFR $<30 \mathrm{ml} / \mathrm{min}$ per $1.73 \mathrm{~m}^{2}$.

The Australian Diabetes Society practice guidelines similarly recommend against metformin with eGFR $<30 \mathrm{ml} / \mathrm{min}$ per $1.73 \mathrm{~m}^{2}$ and caution with eGFR $30-45 \mathrm{ml} / \mathrm{min}$ per $1.73 \mathrm{~m}^{2}{ }^{[6]}$. Thus , although there is clear recognition that renal failure may be a risk factor for adverse events with metformin use there is significant divergence inopinion across the globe regarding the optimal definition of safety. ${ }^{[5]}$

Given the current contraindications, we might consider it a challenge to conduct a new clinical trial to evaluate the use of metformin in individuals with various degrees of impaired renal function,taking into account new criteria for assessing glomerular filtration,attempting to translate creatinine into corresponding eGFR cut points in the context of metformin therapy. The present study was conducted in 102 patients with Type 2 diabetes mellitus with creatnine clearance above $20 \mathrm{ml} / \mathrm{min}$ receiving metformin as monotherapy who attended medical OP or admitted in medical wards in Govt.T D Medical college,Alappuzha in age group 13 years or more. Out of the 102 patients studied, 65 were males and 37 were females. 8 of them were <40 years, 62 were between 40-60 years age group and 32 were more than 60 years age group. The data analysed showed four cases of metabolic acidosis. The bicarbonate levels were low 21.8,21.0,21.4,21.5 $\mathrm{mmol} / \mathrm{L}$ and the eGFR values were $22.50,28.56,23.33$ and $31.27 \mathrm{ml} / \mathrm{min}$ per $\mathrm{m}^{2}$ respectively.

A chi-square test was done to determine the association between the two categorical variables(eGFR and bicarbonate level)in the sample and therefore it is likely to reflect a real association between these 2 variables in the population.Pearson chi-square value was 75.727 with an associated $\mathrm{p}$ value less than 0.001 . The null hypothesis is rejected,since $\mathrm{p}<0.001$ and a conclusion is made that there is a strong dependency existing between GFR and incidence of lactic acidosis in patients on metformin therapy.

\section{Conclusions}

Metformin itself is not a nephrotoxic drug and it is not metabolized in kidney. It is eliminated unchanged by rapid renal excretion through glomerular filtration and possibly by tubular secretion.Impaired renal function decreases renal clearance and may cause accumulation of metformin which increases concerns regarding lactic acidosis.

Metformin can be continued or initiated when the eGFR $<60 \mathrm{ml} / \mathrm{min}$ per $1.73 \mathrm{~m}^{2}$ but the renal function should be closely monitored(every 3-6months).No dose adjustment for metformin is required at eGFR between $45-60 \mathrm{ml} / \mathrm{min}$ per $1.73 \mathrm{~m}^{2}$. The dose of metformin should be reviewed and reduced in patients with eGFR $<45 \mathrm{ml} / \mathrm{min}$ per $\mathrm{m}^{2}$ (eg;by $50 \%$ or to half-maximal dose)and renal function should be monitored closely(every 3 months). Metformin use is cautioned and very close monitoring is required when the eGFR falls to $<30$ and $\geq 20 \mathrm{ml} / \mathrm{min}$ per $1.73 \mathrm{~m}^{2}$. Metformin is not recommended or to be stopped when eGFR falls to $<20 \mathrm{ml} / \mathrm{min}$ per $1.73 \mathrm{~m}^{2}$ 


\section{Suggestions}

1. Further studies involving larger sample size or population based studies may be needed to extend the results for population benefits.

\section{References}

[1]. Pepper GM,Schwartz $\mathrm{M}$ et al ;Lactic acidosis associated with Glucophage use in a ma with normal renal and hepatic function.Diabetes Care 1997 20:232-233

[2]. Stades AME,Heikens JT,Erkelens DW et al;Metformin and lactic acidosis :cause or coincidence? A review of case reports. J Int Med 2004 255:179-187

[3]. Lalau JD ${ }^{1}$.et al Lactic acidosis induced by metformin: incidence, management and prevention. Drug Saf. 2010 Sep 1;33(9):727-40. doi: 10.2165/11536790

[4]. Silvio E. Inzucchi, MD, Kasia J. Lipska, MD, MHS, Helen Mayo,et al Metformin in Patients With Type 2 Diabetes and Kidney Disease .JAMA. 2014 December 24; 312(24): 2668-2675.

[5]. Kasia J. Lipska,,Clifford J. Bailey, PHD, FRCP,Silvio E. Inzucchi, MD et al ; Use of Metformin in the Setting of Mild-to-Moderate Renal Insufficiency .Diabetes Care 2011 Jun; 34(6): 1431-1437

[6]. Rocha A, Almeida M, Santos J, Carvalho A et al;Metformin in patients with chronic kidney disease: strengths and weaknesses. Journal of Nephrology ,01 Jan 2013, 26(1):55-60. 